\title{
Masculinidad y paternidad en procesos de crianza en Medellín, Colombia, 2018*
}

\author{
Masculinity and paternity in parenting processes in Medellín, Colombia, 2018 \\ Masculinidade e paternidade em processos de criação dos filhos em Medellín, \\ Colômbia, 2018
}

\begin{abstract}
Norman Hernando Pérez Rivera'; Mónica Yazmín Giraldo Osorio²; Iván Felipe Muñoz Echeverri
1 Magíster en Educación y Desarrollo Humano. Corporación Universitaria Minuto de Dios, Bello, Colombia. norman.perez@udea.edu. co. ORCID: https://orcid.org/0000-0002-5637-0380

2 Magíster en Educación y Desarrollo Humano. Universidad de Antioquia. Medellín, Colombia. mkgiraldoosorio@gmail.com. ORCID: https://orcid.org/0000-0001-6470-9666

3 Doctor en Salud Pública. Universidad de Antioquia. Medellín, Colombia. ivan.munoz@udea.edu.co. ORCID: https://orcid.org/00000003-3658-432X
\end{abstract}

Recibido: 01/12/2020. Aprobado: 26/10/2021. Publicado: 30/11/2021

Pérez-Rivera NH, Giraldo-Osorio MY, Muñoz-Echeverri IF. Masculinidad y paternidad en procesos de crianza en Medellín, Colombia, 2018. Rev. Fac. Nac. Salud Pública. 2022;40(1):e344529. Dor: https://doi.org/10.17533/udea. rfnsp.e344529

\section{Resumen}

Objetivo: Presentar y discutir significados y prácticas sobre la paternidad y la masculinidad que se dan en la crianza, y que conducen a la reproducción social de los patrones hegemónicos patriarcales o a la emergencia de formas alternativas de ser hombre en la sociedad actual. Metodologia: Estudio cualitativo con base teórica en el interaccionismo simbólico; el procesamiento y el análisis de la información se efectuaron bajo premisas de la teoría fundamentada. Participaron padres y madres de una institución educativa pública de Medellín; se realizaron 6 entrevistas y un grupo de discusión. Resultados: Se describen significados y prácticas sobre masculinidades y paternidades que reproducen el patriarcado en el escenario de la crianza, así como otros que tienden hacia formas alternativas o colaborativas de masculinidad. Igualmente, se describen impulsores para el tránsito hacia masculinidades y paternidades colaborativas en el escenario de la crianza. Conclusión: La crianza posee un lugar determinante en la reproducción del patriarcado, pero engendra, a la vez, la potencialidad de propiciar transformaciones necesarias a favor de masculinidades y paternidades comprometidas con la equidad de género.

--------Palabras clave: Androcentrismo, construcción social del género, crianza del niño, masculinidad, paternidad.

* Este artículo es producto del trabajo de grado para optar al título de magíster en Educación y Desarrollo Humano, del convenio Universidad de Manizales-CINDE (Sabaneta-Antioquia-Colombia): "Significados y prácticas acerca de la masculinidad en el contexto de la crianza, Medellín, 2017-2018". Investigación desarrollada entre octubre de 2017 y noviembre de 2018. 


\begin{abstract}
Objective: To present and to discuss denotations and practices about parenthood and masculinity that occur in parenting, and that lead to the social reproduction of patriarchal hegemonic patterns or the emergence of alternative ways of being a man in today's society. Methodology: A qualitative study theoretically based on symbolic interactionism; information processing and analysis were carried out with a grounded theory approach. Parents from a public school in Medellín were engaged in the study; 6 interviews and a discussion group were conducted.
\end{abstract}

Results: Denotations and practices about masculinities and parenting that reproduce patriarchy were described, and others that tend toward alternative or collaborative forms of parenting were described as well. Likewise, promoters of the transition to masculinities and collaborative parenting are described. Conclusion: Parenting has a decisive role in perpetuating patriarchy, but at the same time, it has the potential to promote necessary transformations in favor of masculinities and parentings committed to gender equality.

-Keywords: Androcentrism, social construction of gender, child rearing, masculinity, parenthood

\section{Resumo}

Objetivo: Apresentar e discutir significados e práticas sobre a paternidade e a masculinidade que se dão na criação dos filhos e que levam à reprodução social dos padrões hegemônicos patriarcais ou à emergência de formas alternativas de ser homem na sociedade atual. Metodologia: Estudo qualitativo com base teórica no interacionismo simbólico; o processamento e a análise da informação foram efetuados sob premissas da teoria fundamentada. Participaram pais e mães de uma instituição educacional pública de Medellín; foram realizadas seis entrevistas e um grupo de discussão. Resultados: São descritos significados e práticas sobre masculinidade e paternidades que reproduzem o patriarcado no cenário da criação dos filhos, assim como outros que se inclinam para as formas alternativas ou colaborativas de masculinidade. Igualmente, são descritos impulsores para o trânsito entre masculinidades e paternidades colaborativas no cenário da criação dos filhos. Conclusão: A criação dos filhos possui um lugar determinante na reprodução do patriarcado, mas gera ao mesmo tempo, a potencialidade de propiciar transformações necessárias a favor de masculinidades e paternidades comprometidas com a igualdade de gênero. ---------Palavras-chave: Androcentrismo; Construção social do gênero; Criação dos filhos; Masculinidade; Paternidade.

\section{Introducción}

Tradicionalmente, las relaciones entre hombres y mujeres han estado moduladas por el patriarcado [1], el cual ha promovido un sistema social desigual a favor del androcentrismo [2], y ha configurado el ser del varón, es decir, su masculinidad, alrededor de aspectos como el éxito económico, la competencia, el ocultamiento de las emociones, la demostración de su virilidad y la promiscuidad $[3,4]$. De esta manera, ha impulsado el abuso del poder o dominación de hombres sobre niñas, niños, mujeres [5], personas de orientación homosexual [6, pp. 167-168] y otros hombres considerados "inferiores" [2, p. 109].

Las diferentes manifestaciones de lucha por la equidad entre géneros, de raíces profundas y extendidas en el tiempo, han evidenciado las graves repercusiones del patriarcado en todas las esferas de la vida social [7]. Así, el orden social patriarcal y las formas hegemónicas de masculinidad han sido reconocidos como responsables de múltiples formas de exclusión social y violencia, tanto a nivel social como doméstico, en contra de las mujeres [8-11]. Más recientemente, se han comenzado a reportar los efectos nocivos en los mismos varones, en cuanto a la desatención de sus problemas de salud [12] y para su salud mental [13, p. 39].

En respuesta a lo anterior, se aprecia, en el panorama global actual, la emergencia de discursos que abogan por la equidad de género, promovidos especialmente por movimientos de mujeres [14] y otros en defensa de los derechos humanos. Algunos de estos discursos han venido ganando gran aceptabilidad social $[15,16]$, e incluso han logrado materializarse en instrumentos jurídicos internacionales y nacionales. Por ejemplo, la Constitución Política colombiana prohíbe cualquier tipo de discriminación [17]; la Ley 581 de 2000 reglamenta la efectiva participación de la mujer en los niveles decisorios de las diferentes ramas y órganos del poder público [18] y la Ley 823 de 2003 busca garantizar la equidad y la igualdad de oportunidades de las mujeres, al establecer porcentajes mínimos de participación de mujeres en estas entidades públicas [19]. A esto se suma la Ley 1257 de 2008, que establece estrategias para afrontar diversas formas de violencia y discriminación contra las mujeres [20].

En esta misma línea, desde hace pocas décadas han comenzado a surgir iniciativas académicas y políticas que pretenden cuestionar las formas hegemónicas de 
masculinidad, oriéntandose más bien hacia significados y prácticas "que buscan redefinir su modo de ser hombres en función de ideas más modernas, que apuestan por el cambio" [21, p. 61]. Estas nuevas manifestaciones de las masculinidades han sido denominadas colaboradoras, "alternativas" [22] o "positivas" [23].

A pesar de ello, las transformaciones hacia la equidad de género en la sociedad son insuficientes, ya que todavía no han logrado concretarse en instrumentos con incidencia clara en espacios de la vida cotidiana [24, pp. 98-101], ni que estimulen a los varones a construir nuevas masculinidades [25] o que transformen eficazmente los patrones culturales predominantes [26,27]. En este sentido, autores en este campo afirman que el patriarcado se ha enraizado históricamente en la estructura social [1,3,5-7], mediante dispositivos económicopolíticos, como los procesos de industrialización y la división sexual del trabajo, que siguen haciendo una marcada separación de roles entre varones y mujeres [3]. La reproducción social del patriarcado también se ha venido dando por mecanismos culturales que reiteran estereotipos de género [13] y patrones de dominación en los diferentes escenarios de socialización, como las interacciones con pares [28], la escuela y la universidad $[29,30] \mathrm{y}$, privilegiadamente, en el entorno familiar y los procesos de crianza [31].

Este manuscrito se ubica en el estudio de esta última vía de reproducción del patriarcado y las masculinidades hegemónicas, lo cual tiene gran importancia debido al carácter prioritario y definitorio de la crianza en los procesos de socialización que experimenta cualquier ser humano [15] y en la aprehensión de los roles de género [22]. En este escenario cobra relevancia la noción de $p a-$ ternidad, entendida como la presencia y el rol del padre en la crianza de los hijos [32, p. 53], que guarda una estrecha relación con la construcción de las masculinidades [32, pp. 53-54], teniendo en cuenta que los padres aportan modelos identitarios de lo que es ser hombre, tanto cuando están presentes como ausentes en este proceso [32, pp. 55-57].

En este sentido, algunas corrientes académicas han argumentado que el rol del padre en la crianza ha ido evolucionando a lo largo del tiempo, pasando de ser guía moral y proveedor económico a adoptar nuevas configuraciones en la actualidad, más integradas en el relacionamiento afectivo con sus hijos e hijas [33, pp. 65-68].

Con todo, son pocas las investigaciones en Colombia que abordan el estudio de las paternidades [34, p. 8] y, especialmente, las interrelaciones entre las formas de paternidad en el proceso de crianza y la emergencia de formas de masculinidad disidentes del modelo patriarcal.

A partir de lo anterior, se formuló el proyecto de investigación: "Significados y prácticas acerca de la masculinidad en el contexto de la crianza, Medellín, 20172018", del que se deriva este artículo, cuyo objetivo es presentar y discutir los principales hallazgos del estudio en cuanto a los significados y las prácticas sobre la paternidad y la masculinidad que se dan en la crianza, y que conducen a la reproducción social de los patrones hegemónicos patriarcales o a la emergencia de formas alternativas de ser hombre en la sociedad actual.

Este trabajo busca contribuir a los esfuerzos académicos y sociales por el resquebrajamiento del patriarcado en la sociedad actual, y aportar insumos, en cuanto al género, para ampliar miradas comprensivas y críticas de análisis e intervención hacia el proceso salud-enfermedad-cuidado y hacia las violencias basadas en género [35], máxime la escasez de investigaciones en el país que captan la voz y subjetividad de los varones sobre estos temas, como señala Galllego-Montes [36, p. 45].

\section{Metodología}

Se realizó una investigación cualitativa, que tomó como soporte epistemológico el construccionismo social [37] y cuya base teórica fue el interaccionismo simbólico [38], el cual entiende la realidad social como el producto de una construcción sociosimbólica, susceptible de ser definida y redefinida por los actores sociales en diferentes escenarios de interacción; asimismo, concibe al ser humano como agente activo y responsable de sus actos.

Metodológicamente, nos apoyamos en los procedimientos básicos de la teoría fundamentada (TF) $[39,40]$, que ha sido utilizada en algunos estudios de género [4143], para analizar y descubrir los significados y las prácticas de los participantes de la investigación respecto al tema de estudio.

La investigación se llevó a cabo en una institución educativa pública que ofrece los grados escolares hasta media técnica, ubicada en la comuna 1 (llamada "Popular") de la ciudad de Medellín. Se contó con la participación de 8 padres y 4 madres de niños, niñas y adolescentes matriculados en dicha institución, quienes se vincularon posterior a un proceso de sensibilización/ socialización que se realizó con la comunidad educativa de la institución mencionada, en el cual se dio a conocer los propósitos, objetivos y procesos metodológicos de la investigación; luego de ello, las personas interesadas en participar se unieron de manera voluntaria.

Los participantes fueron adultos jóvenes entre los 18 y 40 años, con diferentes ocupaciones: conductor de taxi, labores del hogar sin remuneración, empleado del sector salud y empleados independientes. El nivel educativo de los participantes variaba entre profesionales, técnicos y bachilleres.

No se tuvo en cuenta alguna tipología familiar específica como criterio de inclusión en el estudio.

Se garantizó la participación informada, libre y voluntaria de los participantes. 
Como técnicas para la construcción de información, se utilizó la entrevista semiestructurada y el grupo de discusión. Previo a la realización de estas técnicas, se llevó a cabo un proceso de familiarización, que tuvo una duración de 3 meses, el cual consistió en un acercamiento a la comunidad para generar reconocimiento y lazos de confianza, por medio de la participación como asistentes en actividades dirigidas a los padres y las madres, que eran programadas por la institución educativa.

En cuanto a las entrevistas, se retomaron las recomendaciones de Guber, quien describe esta técnica como "una estrategia para hacer que la gente hable sobre lo que sabe, piensa y cree, es una situación en la cual una persona (el investigador-entrevistador) obtiene información sobre algo interrogando a otra persona (entrevistado, informante)" [44, p. 45]. Se llevaron a cabo dos ciclos de entrevistas: en el primero se efectuaron 4 , y en el segundo, 2 (véase Tabla 1).

Para el primer ciclo de entrevistas, se elaboró un guion basado en los objetivos de la investigación, que contaba con tres ejes de indagación: 1) significados y prácticas patriarcales; 2) masculinidades alternativas, y $3)$ prácticas de crianza por parte de padres. La información obtenida fue analizada y se hicieron ajustes al guion para el segundo ciclo, de acuerdo con la saturación de las categorías que emergieron y los lineamientos de muestreo teórico que dicta la TF [45]. Las entrevistas fueron grabadas en formato audio y posteriormente transcritas de manera literal.

Se realizó también un grupo de discusión entre los dos ciclos de entrevistas, en el que participaron 12 personas. La finalidad de este grupo fue profundizar en los hallazgos tras llevar a cabo las primeras entrevistas. Este grupo fue primero audiograbado y después transcrito, para su posterior análisis.

Como premisa analítica, durante el proceso de investigación asumimos una posición de "no conocimiento", con el fin de permitir que los significados, las valoraciones y las descripciones emergieran de manera legítima [46, p. 13].

Tabla 1. Información general de las personas entrevistadas.

\begin{tabular}{cccc}
\hline Entrevista & Edad (años) & Sexo & Estado civil \\
\hline 1 & 35 & & Casado \\
2 & 27 & Masculino & Unión libre \\
3 & 39 & & Casado \\
4 & 28 & & Soltero \\
5 & 32 & Femenino & Casada \\
6 & 25 & Unión libre \\
\hline
\end{tabular}

Para el análisis de información, se tuvieron en cuenta los procesos analíticos propios de la TF [45]. En primer lugar, se realizó la codificación abierta de las entrevistas y de la relatoría del grupo de discusión, de las cuales emergieron las siguientes categorías descriptivas: 1) "crianza", 2) "paternidad", 3) "género", 4) "masculinidades alternativas" y 5) "discursos sociales"; estas se diferenciaron en el análisis de los relatos de los y las participantes. Posteriormente, se desarrolló la codificación axial, mediante la comparación constante y la identificación de propiedades y dimensiones de las categorías emergentes. Finalmente, los hallazgos se organizaron en las categorías analíticas que son descritas en los "Resultados".

La información recolectada se registró y sistematizó por medio de diarios de campo, grabaciones de audio, mapas conceptuales y fichas de contenido. Para esto se utilizaron los software: Microsoft Word ${ }^{\circledR}$ 2010, Atlas-ti versión 8.0, cuya licencia era propiedad de los investigadores, y el software libre CMap Tools versión 6.01.01.

Los fundamentos éticos que dieron soporte a la investigación se basaron en el reconocimiento y el respeto de la subjetividad de los participantes, a partir de la valoración y la protección de los derechos y la dignidad humana. Esto implicó estas consideraciones: 1) permitir que los participantes cambiaran de opinión y que pudieran retirarse sin sanción de ningún tipo; 2) la reserva en el manejo de la información fue respetada, con reglas explícitas de confidencialidad; 3) la información que se produjo en la investigación fue dada a conocer a los participantes al concluir el estudio; y 4) el bienestar de los participantes se vigiló cuidadosamente a lo largo del proceso [47].

Estas consideraciones fueron socializadas a los participantes durante el proceso de familiarización antes descrito, y se solicitó a cada uno la firma de un documento de consentimiento informado. El correspondiente 
consentimiento informado para la realización del grupo de discusión se tomó de manera verbal.

Adicionalmente, y con el fin de proteger las identidades de los participantes, se usaron nombres falsos para hacer referencia a los entrevistados y participantes del grupo de discusión.

Se realizó un taller reflexivo, como mecanismo para hacer devolución a los participantes sobre los resultados del proceso investigativo y los aprendizajes que de él se derivaron.

\section{Resultados}

Según los participantes, hay estrechas relaciones entre la configuración de la masculinidad y las experiencias vivenciadas en la crianza (véase Figura 1). Estas relaciones están mediadas por la lectura que los hijos y las hijas hacen de las prácticas masculinas y paternales en su familia (sin perjuicio de las prácticas femeninas y maternales, que no fueron estudiadas a fondo en este trabajo), y de las formas de interacción y el vínculo entre el padre y los hijos e hijas. Por ejemplo, para los varones - afir- man-, lo vivido con su padre ha sido fundamental en el proceso de constituirse como tales.

De acuerdo con los participantes, los niños y las niñas configuran sus roles de género en el escenario de la crianza - los cuales reproducen cuando asumen el lugar de padres o madres en el futuro-, como puede apreciarse en los siguientes fragmentos de entrevista:

Se aprende a ser hombre por lo que hablábamos ahorita: la casa, el legado, la enseñanza de un papá presente; un papá activo en su función a ser papá enseña a ser hijo a ser hombre (José, 39 años).

Para mí, ser hombre es ser una persona responsable, respetuosa, cumplir con sus obligaciones, como le inculcaron a uno desde pequeño [...] eso lo inculcan a uno desde el hogar, desde el padre [...], uno desde el hogar aprende a ser hombre (Antonio, 28 años).

En este orden de ideas, el estudio realizado permitió discriminar significados y prácticas respecto a las masculinidades y la paternidad en el escenario de la crianza, que apuntan a la reproducción de masculinidades patriarcales hegemónicas; pero también, otras que tienden

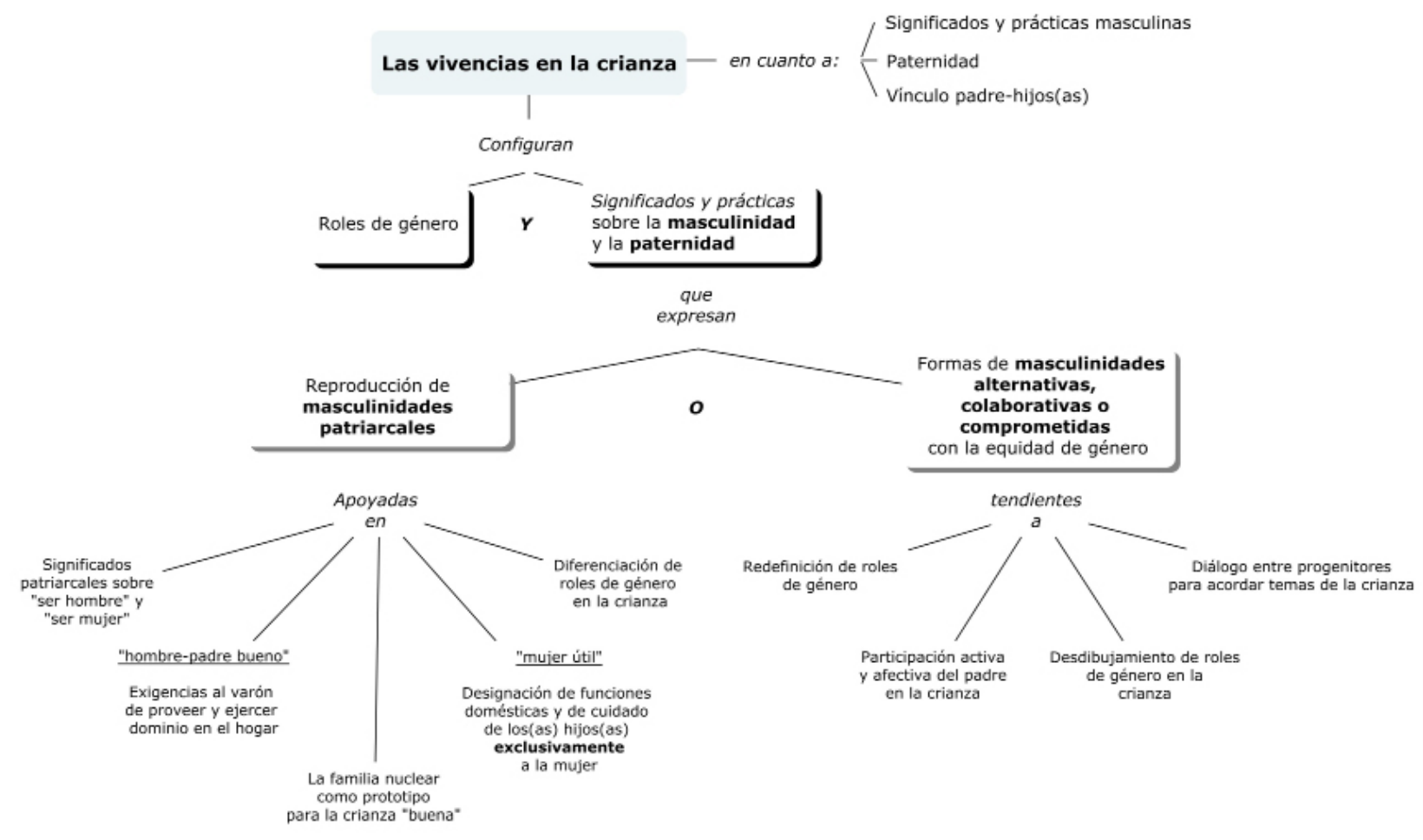

Figura 1. Síntesis de hallazgos acerca de significados y prácticas sobre masculinidad y paternidad en procesos de crianza referidos por los participantes. 
a desplazar las masculinidades a favor de formas alternativas, colaborativas o comprometidas con la equidad de géneros. A continuación se describen los hallazgos al respecto, comenzando por aquellos que reproducen el modelo patriarcal.

\section{Masculinidades y paternidades patriarcales en el escenario de la crianza}

Se evidenció que la crianza se constituye en un escenario de socialización clave para la reproducción de significados y prácticas patriarcales.

Tras analizar lo aportado por los participantes, el discurso patriarcal se justifica en el supuesto desequilibrio de poder entre hombres y mujeres, y se basa en las siguientes concepciones respecto a "ser hombres": 1) los hombres se desenvuelven prevalentemente en el ámbito público; 2) son polígamos; 3) son herederos de la hegemonía masculina, y 4) son los llamados a ejercer la autoridad en diferentes escenarios, como la familia, el trabajo y la sociedad en general.

Estas características exigen a los hombres-padres a posicionar un rol dominante en la crianza, bajo la premisa de brindar "estabilidad" al hogar, y les otorga el "derecho" a ejercer un tipo de soberanía "especial" sobre los demás miembros del hogar. En este sentido, un participante afirmaba:

Si la mujer fuese más sabia a la hora de dar una respuesta, de pronto muchos pleitos no se darían, porque el hombre, al ser hombre, tiene algo de machista, y el hombre no quiere que nadie le hable duro (José, 39 años).

Esta perspectiva da lugar al concepto planteado por los participantes de "hombre bueno", correspondiente a aquel que, además de cumplir la función de proveer las necesidades básicas y brindar protección a los miembros de la familia - lo que es entendido por los participantes como ser "guía" de su familia_, reafirma su rol mediante el ejercicio autoritario del poder: "en mi casa, la autoridad la ejerzo yo, y reconozco que soy autoritario" (José, 39 años).

Para los entrevistados, a través de la crianza, los niños incorporan que no basta con "aprender" a ser hombres, sino que adquieren el principio moral de ser "buenos", que implica las funciones de provisión, protección y ejercicio de la autoridad.

Así, de estas concepciones patriarcales de la masculinidad se desprenden orientaciones como la distribución de roles diferenciales entre niños y niñas, y la idea de que la crianza de los hijos varones debe tener mayor exigencia, dada la autoridad que heredarán, a fin de que puedan ejercer de manera exitosa el legado del padre en el futuro.

Por otro lado, los discursos y las prácticas en torno al ser femenino circunscriben su rol en la vida de pa- reja, las labores domésticas y la crianza, subordinando este ser a la autoridad masculina. En este sentido, para algunos participantes, varones y mujeres, la mujer tiene las funciones de cuidar, educar y realizar las labores de casa, siendo así la figura principal en la crianza y la responsable de los oficios domésticos.

Entrevistados afirman que si una mujer cumple bien sus funciones a partir de lo asignado socialmente, y se acoge a la autoridad del hombre, será exitosa en su hogar, como lo manifiesta José (39 años):

Por ejemplo, por mi hija, yo le digo a mi esposa: “enséñale a hacer sopita $[\ldots]$ que nos haga el desayuno tal día". Empezamos a jugar ese juego de roles, porque yo le digo a mi hija: "Mire, que su esposo me diga a mí: 'gracias suegro, porque me entrego una mujer útil"'.

Estas afirmaciones recaen en el adjetivo mujer "útil", usado por algunos participantes para referirse a quien cuida de los hijos - y del esposo-, además de ser quien permanece en casa y lleva a cabo las labores domésticas.

Desde esta perspectiva, la equidad entre hombres y mujeres consiste en la posibilidad, para la mujer, de trabajar también fuera de casa, con lo que puede contribuir económicamente para el sustento del hogar. Esta condición le confiere un rol diferente al interior del hogar, ya que la provisión económica le faculta también para aportar ideas. Sin embargo, mujeres y varones participantes coinciden en que esto no la exime de ser la responsable directa de la crianza y el cuidado de los hijos e hijas.

Aunado a lo anterior, se aprecia en el discurso de varones y mujeres que las familias adecuadas para la crianza son aquellas donde prima la autoridad masculina y tienen una composición nuclear — es decir, que está constituida por padre y madre-. De este modo, una familia con esta tipología recibe, per se, el atributo de ser el nicho para alcanzar una "buena" crianza:

De pronto del hogar donde la persona se está levantando, que sea una persona que también se levante con papá y mamá, que eso influye muchísimo en los niños (Pedro, 35 años).

Según algunos participantes, ciertos preceptos religiosos explican y dan fundamento a estas concepciones sobre la masculinidad y la familia nuclear, ya que, para algunos entrevistados, la figura de Dios es asimilada como el gran padre, que otorga a los hombres la paternidad, a quienes, además, orienta para una buena crianza.

\section{Masculinidades y paternidades colaborativas en el escenario de la crianza}

Aunque, en el discurso de los participantes, se evidenció la fuerte presencia del patriarcado en el escenario de la crianza, los hallazgos también muestran algunos significados y prácticas que toman distancia de esta perspecti- 
va y se mueven - mudan - hacia formas alternativas de las masculinidades y de relacionamientos entre hombres y mujeres en el ámbito familiar.

En este orden de ideas, aunque el discurso predominante antes descrito distingue radicalmente los roles para hombres y mujeres, se identificaron algunas tendencias que apuntan a redefinirlos y transformarlos. Así, hubo referencias sobre algunas familias en las cuales existen acciones colaborativas, que dejan entrever prácticas y significados que divergen del patrón patriarcal. Tal es el caso de los oficios domésticos, que son compartidos como signo de cooperación en el hogar y que no son catalogados por los hombres como riesgo para su masculinidad:

Pues el hecho de ser hombre encierra muchas cosas, pero el serlo no significa ser machista, que es lo que de pronto no sabían diferenciar anteriormente; yo no dejo de ser hombre por colaborarle a mi esposa en los oficios de la casa (Pedro, 35 años).

También se identificaron prácticas en las que el padre y la madre participan activamente en la crianza. Esto se traduce en que el cuidado, el acompañamiento y la educación de los hijos e hijas dejan de ser rol exclusivo de la mujer y pasa a ser tarea compartida por ambos progenitores. Asimismo, se encontraron expresiones que evidencian que la figura del ejercicio autoritario de la paternidad es permeada por prácticas de diálogo y expresiones de afecto entre padres e hijos e hijas, y entre padre y madre.

Ahora bien, aunque el padre no renuncia a su rol como proveedor de bienes materiales, se aprecia una cierta relativización de su obligación exclusiva en la provisión económica, dado el importante aporte que asumen las madres en la manutención familiar. Llama aún más la atención que, en algunos casos, la provisión económica no concede directamente el rol de autoridad familiar al padre, sino que va surgiendo la necesidad, tanto para las mujeres entrevistadas como para algunos varones, de que existan acuerdos consensuados respecto al ejercicio de la norma y la autoridad en el hogar. En este sentido, emergen expresiones que hacen referencia a la negociación de responsabilidades y de criterios para el establecimiento de las normas y el ejercicio de la autoridad en el proceso de la crianza, a la par que se da libertad para manifestaciones afectivas de ambos progenitores hacia sus hijos e hijas. En estas familias, se nota algún grado de desdibujamiento de los roles, permitiendo a padre y madre, tanto el ejercicio de la autoridad como la expresión del amor y los afectos, en el acompañamiento y la crianza de sus hijos e hijas.

Adicionalmente, los padres logran propiciar ambientes de confianza con sus hijos e hijas, por medio del acompañamiento en asuntos importantes de la crianza, como la disciplina, la responsabilidad y el cuidado de sí.
Asimismo, motivar el disfrute de momentos en familia, lo que posibilita también que la crianza para niños y niñas no tenga diferenciaciones basadas en el género:

Con mi esposa e hija, la autoridad se da más como por igual. En cuanto a la niña y en cuanto a la toma de decisiones, es algo más consensuado. Las demostraciones de cariño se dan abiertamente y se dan a conocer sin temores, a través de detalles, de palabras y de celebraciones (Lucas, 27 años).

\section{Impulsores para el tránsito hacia masculinidades y paternidades colaborativas en el escenario de la crianza}

Siguiendo lo mencionado por los participantes, el proceso de mudanza hacia las masculinidades y paternidades alternativas, colaborativas o comprometidas con la equidad de géneros no es fácil. Aunque se identifican algunos asuntos que favorecen el cambio, también se mencionan obstáculos que les han impedido una apropiación mayor de las formas alternativas de masculinidad.

Un elemento que resalta en los hallazgos es el hecho que los discursos sociales sobre la equidad de género han logrado, de algún modo, condicionar las maneras en que los hombres perciben y se relacionan con las mujeres, promoviendo un mayor respeto y valoración de las mujeres por parte de los hombres, frente a los diferentes roles que estas desempeñan dentro y fuera del hogar:

Teniendo presente la libertad que hay ahora para la mujer, pues para mí es algo muy valioso que nosotros como hombres debemos respetarlas y valorarlas, ante todo en sus trabajos, pues muchas veces les toca afrontar el papel de papá y mamá; es la figura para querer, respetar y valorar (Pedro, 35 años).

Por otro lado, se evidenció que, para algunos padres, el ejemplo recibido de una madre cabeza de hogar se convirtió en un factor que incide de manera positiva en la perspectiva que tienen ahora de las mujeres, pues el vivenciar la destreza de su propia madre al haberle criado, en muchos casos sin el apoyo de un padre, los lleva a mayores niveles de respeto y una valoración muy positiva hacia las mujeres.

También el ser padre de una hija les permite a los varones resignificar su rol masculino y los lleva a modificar sus prácticas de crianza. De esta manera, les motiva a propiciar ambientes de equidad entre géneros y evitar la reproducción de prácticas desfavorables para las mujeres:

Yo, como hombre, he cambiado mucho la mentalidad, porque también la niña me ayudó a cambiarla; si antes veía [a] las mujeres de cierto modo, al tener una hija, lo veo de manera diferente, porque yo no quiero que ella se enfrente al mundo como tal vez muchas mujeres se enfrentaron anteriormente (Lucas, 27 años) 
Para algunos padres, la participación activa en la crianza de sus hijos e hijas se convierte en una motivación vital y un estímulo para trazarse metas de superación personal, lo cual, a su vez, ha generado transformaciones en la perspectiva del cómo se relacionan con los otros, las otras y consigo mismos. Pues aquellos padres que han asumido la crianza de manera activa perciben en ello un valor agregado que no hubiese sido posible sin ese acontecimiento, como lo expresa el siguiente relato:

[...] y yo por ella hago todo, porque cuando yo no tenía a Sofía, a mí no me gustaba estudiar. [...] Y desde que Sofía nació, ¡hijuemadre!, por ella me tengo que pelar [esforzar] (Antonio, 28 años).

No obstante, para los varones y las mujeres entrevistados, persiste un gran obstáculo para lograr el tránsito hacia las masculinidades alternativas, y es el temor de algunos hombres a perder el estatus socialmente dado, lo que implicaría la reconfiguración de su rol y la pérdida de sus privilegios patriarcales. En este sentido, el progresivo empoderamiento de las mujeres en la esfera pública, gracias a trabajar y estudiar fuera del hogar, es visto por algunos varones como una amenaza, en tanto ha llevado a algunas mujeres a cuestionar la noción de mujer "útil", afín a la lógica patriarcal.

Siguiendo esta línea, algunos varones y mujeres entrevistados temen las transformaciones familiares y en la crianza, derivadas de la salida femenina del ámbito doméstico, ya que las actividades de la crianza y las labores domésticas pasan a ser desempeñadas por otros, a ser delegadas al padre, o incluso, a que este quede en último plano, lo que, según ellos, desestabiliza el patrón tradicional del hogar y genera cambios, con posibles resultados desfavorables en el bienestar de los hijos e hijas, y para las relaciones familiares.

Al temor mencionado, se suma la inseguridad de los varones por no saber cómo asumir estos nuevos roles, ya que no le fueron enseñados en su crianza ni en otros escenarios de socialización.

Por otro lado, los participantes manifiestan que a nivel de la sociedad en general siguen teniendo un gran peso las prácticas y los discursos que favorecen la reproducción del patriarcado, por ejemplo, en productos de los medios de comunicación (novelas, noticias, series de televisión, entre otros), por lo que se constituyen como otro obstáculo que les impide una mudanza más decidida hacia masculinidades alternativas. Lo anterior se expresa también en la censura que se percibe a nivel comunitario, frente a las posturas de quienes no asumen los roles predefinidos ni replican las fórmulas patriarcales en la crianza.

\section{Discusión}

Este trabajo presenta una mirada al problema del patriarcado desde el escenario de socialización de la crianza, entendida esta como una de sus principales vías de reproducción $[9,15,31,32]$. Se optó por privilegiar el punto de vista de los varones y del rol paterno, dada la escasez de investigaciones al respecto [36]; incluso, aunque se contó con voces de mujeres, la indagación se orientó hacia el ejercicio de la paternidad y las masculinidades.

Lo anteriormente expuesto deja ver las limitaciones de este estudio para una comprensión integral del fenómeno de la reproducción del patriarcado en nuestra sociedad, dado que no se ahondó en el rol de la maternidad, ni de la relación padre-madre, ni en la voz de las hijas y los hijos.

Asimismo, dada la configuración del grupo de entrevistados, los hallazgos y la discusión de los mismos se centran en el criterio de personas adultas jóvenes, lo que influye en los análisis efectuados, teniendo en cuenta alguna evidencia que indica que la edad influye en las posiciones respecto a los asuntos de género, las masculinidades y la paternidad [48; 49, pp. 47-53; 50, pp. 22-34]. Por ejemplo, un estudio de tipo cuantitativo realizado en Chile en 2016, indicó una mayor tolerancia y democratización de roles en jóvenes de entre 18 a 29 años, lo cual permite inferir una concepción de equidad de género que va en aumento por parte de las nuevas generaciones, en comparación con las influenciadas por el modelo tradicional de masculinidad [51].

Desde el alcance explicitado de esta investigación, destacamos que el análisis de los hallazgos evidencia que en el escenario de la crianza hay significados y prácticas patriarcales que son reproducidos por hombres y mujeres; sin embargo, también se identificaron formas alternativas de significar a los hombres y a los padres, que apuntan a una mayor equidad entre géneros y posibilitan la configuración de masculinidades alternativas en sus hijos.

En cuanto a lo descrito respecto a la pervivencia de significados y prácticas patriarcales, Álvarez [49] señala que, efectivamente, aún persiste una defensa del patriarcado en la sociedad, que se da de manera sutil y generalizada entre los mismos varones y también por las mujeres. El mismo autor y otros más citados afirman que las prácticas y los discursos hegemónicos se ven representados en diferentes escenarios sociales, lo que lleva a que se perpetúen por medio de los discursos institucionales de la familia, la escuela y la religión [49, pp. 35-44; 50, pp. 53-107; 51, pp. 37-38; 52, p. 57]. 
El patriarcado tradicionalmente ha separado el mundo en dos esferas, debido a una distribución estricta de actividades, espacios y herramientas para cada uno de los dos sexos, con la oposición entre lo público y lo privado. De esta manera, lo privado es feminizado, se desarrolla en el ámbito familiar, a la que fueron confinadas las mujeres; y lo público, donde se desarrollan las actividades productivas, sociales y políticas, es masculinizado [1;31, pp. 17; 53, pp. 7-12].

Desde esta forma de estructuración social, reproducida de manera hegemónica por siglos, la crianza de los hijos e hijas podría entenderse como un asunto de responsabilidad materna, circunscribiendo la labor paternal a la provisión de los bienes materiales y al ejercicio autoritario de la norma. Es decir, la paternidad se convierte en un reflejo del ejercicio de la masculinidad patriarcal [24, pp. 98-99; 54, pp. 40-41].

Siguiendo en esta línea, los adjetivos de "bueno" y "útil", atribuidos a hombres y mujeres respectivamente por los entrevistados, implican que el ser "hombre bueno" prácticamente exime al hombre-padre de tareas y funciones relacionales en la crianza, más allá de la provisión de bienes y el ejercicio autoritario o dictatorial de la norma. En cambio, para la mujer, el adjetivo "útil" remite a un imperativo por prácticas domésticas y relacionales confinadas a la esfera privada, con exigencia de calidad, lo que hace evidente el gran peso de responsabilidad que recae en las mujeres-madres. Es decir, los significados y las prácticas patriarcales que fueron identificados, y que coinciden con algunos referentes bibliográficos citados, reproducen patrones en los que la dinámica "ideal" para la crianza se desarrolla cuando los hombres son "buenos" al desenvolverse en la esfera pública, y las mujeres son "útiles" en el ámbito privado, a partir de la sumisión a la autoridad masculina y la "buena" realización de las tareas domésticas y el cuidado de los hijos [2, pp. 105-107; 9; 24 , pp. $98-99 ; 25 ; 31$, p. 20, 52].

Estos adjetivos de "bueno" y "útil" contribuyen también a la ampliación de las brechas de desigualdad e inequidad entre géneros. En este sentido, los hallazgos igualmente sugieren que las prácticas de crianza basadas en la díada "hombre bueno" / "mujer útil", en especial cuando son desplegadas en una estructura familiar nuclear, conducen a criar "buenos" hombres y mujeres "útiles", quienes a su vez perpetuarían los roles que fundamentan el patriarcado.

Precisamente, en cuanto a la estructura familiar nuclear como la organización prototípica para la buena crianza, los hallazgos revelan el fuerte influjo de la cultura occidental, que modela los significados y las prácticas de hombres y mujeres, padres y madres, a través de las instituciones sociales como la familia, la escuela, la Iglesia, entre otras, y que en todas las culturas se trasmite de generación en generación [55-57].
No obstante, a pesar del fuerte influjo patriarcal, esta investigación logra identificar algunas expresiones divergentes al patrón hegemónico ya discutido, las que pueden explicarse a partir de los postulados socioconstruccionistas del género, sustentadas en el argumento acerca de las masculinidades como construcción histórica, social y cultural $[1,3,58]$, según las cuales estas configuraciones no son estáticas, ni son las mismas siempre y en todas partes [21, pp. 23-24; 56, pp. 68-85]. De acuerdo con esto, los autores de este artículo comprendemos estos hallazgos como oportunidades de resquebrajamiento del patriarcado a favor de manifestaciones alternativas de masculinidades y paternidades.

Así como se ha señalado la relación entre el patriarcado, las masculinidades hegemónicas y las paternidades basadas en el autoritarismo y la provisión material, podría también hablarse de la interconexión entre las masculinidades comprometidas con la equidad de géneros y paternidades "activas" [25], "positivas" [32] o "presentes" [2, p. 116], cuyas características son estar comprometidos en las labores de cuidado [31, p. 35] e involucradas afectivamente y vinculadas de manera democrática en la crianza [22].

Para el caso de esta investigación, se observaron prácticas de crianza "alternativas", en las que los niños adquieren elementos para confrontar el molde patriarcal a favor de la equidad de género y el respeto hacia los otros y las otras.

A pesar de lo anterior, apreciamos que el posicionamiento de estos significados y prácticas contrahegemónicos aún es incipiente, indicando que la mudanza hacia la equidad de género está en proceso. Al respecto, Álvarez [49, p. 83] afirma que existen prácticas que son más fáciles de modificar que otras, algunas identificadas en esta investigación, como la participación de las mujeres en mercado laboral y la distribución cooperativa de las tareas domésticas. Empero, resultan más difíciles de afrontar los matices y las particularidades más relevantes del discurso de la hegemonía masculina.

En este mismo orden de ideas, Mazo [55, p. 56] ha señalado que aunque los varones hablen con aparente convencimiento de la equidad de género y la llamada "liberación femenina" — como lo escuchamos en algunos participantes-, su discurso y su hacer son contradictorios; tal es el caso de las afirmaciones en las que los mismos participantes exaltan su participación en las tareas domésticas como un gran paso en cuanto a la equidad de género, pero al usar términos como "colaborarle a ella" o "ayudarle", siguen mostrando aún mucha dificultad en la inserción masculina en la crianza de los hijos y reafirman así que la responsabilidad doméstica recae en sus parejas femeninas. Siguiendo esta línea, Muñoz cuestiona que estos son cambios que se han dado "más por ser políticamente correctos que por una transformación de imaginarios" [14, p. 22]. 
Estudios más recientes sugieren otras ópticas sobre el asunto, al referirse a la coexistencia, en la sociedad, de formas compatibles de masculinidad hegemónica con otros tipos [51, p. 180]. Asimismo, en el campo de la crianza, se ha acuñado la noción de paternidades paradójicas, que alude a formas de paternar que combinan elementos tradicionales con formas ligadas a paternidades activas, presentes o comprometidas (como se describieron arriba) [22, p. 116]. Estas perspectivas aportan elementos adicionales para la comprensión de algunos hallazgos, en tanto invitan a la reflexión sobre el problema del patriarcado, las masculinidades y las paternidades no como una bipolaridad hegemónico/alternativo, sino como diversas vías mediante las cuales las relaciones desiguales de poder entre géneros buscan su transformación, en muchos casos, hacia modos más democráticos y humanizantes.

En este sentido, este trabajo presenta algunos aspectos que posibilitan, y otras que limitan, nuevos caminos hacia la equidad de género. En primer lugar, y en coincidencia con algunos referentes bibliográficos, las nuevas generaciones son más permeables para la adopción de modelos alternativos de masculinidad y a las otras maneras alternas de ser hombre [21, p. 98;48; 49, pp. 47-53; 50, pp. 22-34; 51]. Al respecto, Álvarez [49, pp. 84-87] plantea que las generaciones jóvenes tienen mayor apertura al cambio, debido a influencias globales y a que conviven con perspectivas emergentes que les permiten aceptar y adaptarse a los cambios propuestos desde los discursos de reciente aparición. Beiras afirma que las nuevas generaciones portan nuevas miradas hacia las relaciones de género y para la paternidad [50].

En segundo lugar, se identificó que el mismo ejercicio de la paternidad es una vía para la transformación de las relaciones desiguales de género, ya que posibilita procesos de resignificación de los estereotipos sobre la autoridad y el vínculo entre padres e hijos e hijas [13, p. 41; 59].

Por otro lado, la presión social y el temor a la sanción social, de una u otra forma evidencian una ruptura o fisura en las masculinidades hegemónicas y permiten la fuga a favor de la equidad de género. Álvarez hace referencia a hallazgos de investigación donde los participantes reconocen que los comentarios y las críticas surgidas de familiares, amigos y conocidos hacia el machismo inhiben las prácticas y las concepciones que reproducen el patriarcado [49, p. 80].

Pero también siguen presentes, como grandes barreras para vencer la reproducción del patriarcado, los estigmas y estereotipos culturales que generan múltiples conflictos y temores para que un padre se atreva a ser un varón "diferente" [13, p. 41], temores que en ocasiones son compartidos por sus parejas mujeres, como vimos en los hallazgos. Otra limitante que ha sido reportada por la literatura y que se destaca acá, a pesar de no ser identificada en los hallazgos, consiste en los obstáculos del mercado laboral y las deficiencias en las políticas públicas para impulsar la equidad de género y una construcción equitativa de los cuidados en la niñez [24,25].

Con lo dicho hasta acá, se concluye que la fuerza del patriarcado no es absoluta - si lo fuera, no se fracturaría - y es por eso por lo que las tensiones sobre el sistema patriarcal ocasionan desdibujamientos en sus prácticas sociales, que se habían instalado como las únicas maneras de relacionarse entre hombres y mujeres. Además, que la crianza es una práctica social y un escenario que favorece la reproducción de masculinidades patriarcales, pero también engendra la potencialidad, la fuerza suficiente, para generar las transformaciones necesarias a favor de la equidad y la convivencia en respeto por la diferencia.

Por tanto, es necesario que la forma de criar sea diferente. Se necesita combatir el machismo en la paternidad y concientizar a los padres en la importancia de su rol y de compartir las tareas con la madre, dado que la implicación de los hombres en la crianza y en los trabajos reproductivos es un factor clave para la transformación de la realidad hacia paradigmas sociales y de relación más justos e igualitarios [32]. Igualmente, se debe retomar que la práctica de paternar "es una excelente puerta de entrada para el trabajo preventivo con hombres en violencia contra las mujeres y niñas, y en violencia en género" [24, p. 101]. Se reafirma acá que el escenario de la crianza es un lugar de transformación y una gran oportunidad para gestar el cambio en la manera de relacionarnos los unos con otros.

Pasar de lo anhelado a lo real implica entender las potencialidades transformadoras de la crianza, teniendo en cuenta el conocimiento de los significados y las prácticas tanto tradicionales y hegemónicas como el de los que invocan nuevas masculinidades. Así, posibilitar los procesos pedagógicos y sociales necesarios para construir las nuevas significaciones y prácticas que buscamos en el futuro cercano, no se da sin un trabajo con hombres y mujeres, que "pasa por la ruptura de los mandatos patriarcales y sus encargos: tener el poder y el control, ser proveedor, ser el guerrero protector y controlador" [60, p. 15].

Finalizamos invitando a hacer conciencia de los propios actos a favor de la equidad de género. Para ello se hace necesario una constante autoobservación, y así, alcanzar el nivel de conciencia necesario y, a su vez, con otros y otras, pensar de manera alternativa respecto a la fuerza de las tradiciones.

\section{Agradecimientos}

Agradecimiento especial a las y los participantes por su entusiasmo para hacer parte de la investigación y los va- 
liosos aportes que nos entregaron. Igualmente, agradecemos a la Maestría en Educación y Desarrollo Humano del convenio Universidad de Manizales-CINDE (Sabaneta-Antioquia-Colombia), por el apoyo académico.

\section{Declaración de fuente de financiación}

Esta investigación fue financiada exclusivamente por los investigadores.

\section{Declaración de conflictos de intereses}

Los autores no tienen ningún conflicto de interés para declarar.

\section{Declaración de responsabilidad}

Los puntos de vista expresados en el manuscrito son de responsabilidad estricta de los autores y no comprometen la institución de afiliación de los autores.

\section{Declaración de contribución por autores}

Norman Hernando Pérez-Rivera: concepción y diseño del estudio, análisis e interpretación de datos, escritura del artículo y aprobación de la versión final del mismo. Se responsabiliza de todos los aspectos del trabajo.

Mónica Yazmín Giraldo-Osorio: concepción y diseño del estudio, análisis e interpretación de datos, escritura del artículo y aprobación de la versión final del mismo. Se responsabiliza de todos los aspectos del trabajo.

Iván Felipe Muñoz-Echeverri: contribuciones sustanciales al diseño del estudio, análisis e interpretación de datos, escritura del artículo y aprobación de la versión final del mismo. Se responsabiliza de todos los aspectos del trabajo.

\section{Referencias}

1. Badinter E. XY, la identidad masculina. Barcelona, Bogotá: Grupo Editorial Norma; 1993.

2. Bard G. Aferrarse o soltar privilegios de género: sobre masculinidades hegemónicas y disidentes. Península. 2016;11(2):101-22. DoI: https://doi.org/10.1016/j.pnsla.2016.08.003

3. Connell R. Masculinidades. $2 .^{a}$ ed. Mexico: Universidad Naciona Autónoma de México, Programa Universitario de Estudios de Género; 2015 .

4. Viveros M. Teorías feministas y estudios sobre varones y masculinidades. Dilemas y desafíos recientes. Revista La Manzana de la Discordia. 2007;2(2):25-36. DoI: https://doi.org/10.25100/ lamanzanadeladiscordia.v2i2.1399

5. Bourdieu P. La dominación masculina. Barcelona: Anagrama; 2006.
6. Bustamante WA. Masculinidad y homofobia. El control de la sexualidad del varón en la construcción del Estado colombiano. Sociedad y Economía. 2013;(24):159-82.

7. Butler J. Mecanismos psíquicos del poder. Teorías sobre la sujeción. Madrid: Cátedra; 2001.

8. Peña JC, Arias LA, Sáez F. Masculinity, socialization and justification of gender violence in men of the region of Araucanía (Chile). Masculinities Soc. Chang. 2017;6(2):142-65. Dor: https://doi. org/http://dx.doi.org/10.17583/mcs.2017.2235

9. Jewkes R, Morrell R, Hearn J, et al. Hegemonic masculinity: Combining theory and practice in gender interventions. Cult Health Sex. 2015;17(Supl. 2):112-27. Dor: https://doi.org/10.1080 /13691058.2015.1085094

10. Kuhl DC, Warner DF, Warner TD. Intimate partner violence risk among victims of youth violence: Are early unions bad, beneficial, or benign? Criminology. 2015;53(3):427-56. Dor: https://doi. org/10.1111/1745-9125.12075

11. García-González DE, Fernández de la Reguera Ahedo A. Trascender la violencia de género desde el reconocimiento ético: un diálogo entre la filosofía y la empiria en contextos migratorios. Universum. 2017;32(1):77-94. Dor: http://dx.doi.org/10.4067/ S0718-23762017000100077

12. Torres-Pagán L, Toro-Alfonso $\dagger \mathrm{J}$. Hegemonic masculinity as a key factor on health beliefs and seeking help in Puerto Rican men with hypertension: A qualitative study. Revista Puertorriqueña de Psicología. 2017;28(1):134-47.

13. Salguero A, Córdoba D, Sapién S. Masculinidad y paternidad: los riesgos en la salud a partir de los aprendizajes de género. Psicología y Salud. 2018;28(1):37-44. Dor: https://doi.org/10.25009/pys. v28i1.2546

14. Muñoz H. Hacerse hombres. La construcción de masculinidades desde las subjetividades. Medellín: Universidad de Antioquia, Fondo Editorial FCSH; 2017.

15. Palacio MC, Valencia AJ. La identidad masculina: un mundo de inclusiones y exclusiones. Manizales: Editorial Universidad de Caldas; 2001.

16. Solano FJ, Mosquera SP. Significados sobre la masculinidad construidos por hombres adultos de Buenaventura. Revista La Manzana de la Discordia. 2018;13(2):73-90. DoI: https://doi. org/10.25100/lamanzanadeladiscordia.v13i2.7161

17. Colombia. Constitución Política de la República de Colombia (1991 julio 20).

18. Colombia. Congreso de la República. Ley 581, por la cual se reglamenta la adecuada y efectiva participación de la mujer en los niveles decisorios de las diferentes ramas y órganos del poder público, de conformidad con los artículos 13,40 y 43 de la Constitución Nacional y se dictan otras disposiciones (2000 mayo 31).

19. Colombia. Congreso de la República. Ley 823, por la cual se dictan normas sobre igualdad de oportunidades para las mujeres (2000 julio 10).

20. Colombia. Congreso de la República. Ley 1257, por la cual se dictan normas de sensibilización, prevención y sanción de formas de violencia y discriminación contra las mujeres, se reforman los Códigos Penal, de Procedimiento Penal, la Ley 294 de 1996 y se dictan otras disposiciones (2008 diciembre 4).

21. Faur E. Masculinidades y desarrollo social: las relaciones de género desde la perspectiva de los hombres. Bogotá: Arango Editores [internet]; 2004 [citado 2021 ago. 3]. Disponible en: https:// www.unicef.org/colombia/media/2376/file/Masculinidades $\% 20$ y\%20Desarrollo\%20Social.pdf 
22. Montenegro JL, Orcasita L, Tunubala L, Zapata L. Representaciones sociales sobre masculinidad y paternidad en padres con hijos gays. Inv Enf. 2019;21(1):1-10. Dor: https://doi.org/10.11144/Javeriana.ie21-1.rsmp

23. Boscán A. Las nuevas masculinidades positivas. Utopía y Prax. Latinoam. [internet]. 2008 [citado 2021 ago. 3]; 13(41):93-106.

24. Aguayo F, Barker G, Kimelman E. Editorial: Paternidad y cuidado en América Latina: ausencias, presencias y transformaciones. Masculinities Soc. Chang. 2016;5(2):98-106. Dor: https://doi. org/10.17583/MCS.2016.2140

25. Guerrero CI, Armstrong Barea L, González Adonis F, Bratz J, et al. Paternidad activa y cuidado en la niñez: reflexiones desde las desigualdades de género y la masculinidad. Enferm. Actual de Costa Rica [internet]. 2020 [citado 2021 mar. 22]; (38):282-91. Disponible en: https://www.scielo.sa.cr/pdf/enfermeria/n38/1409. 4568-enfermeria-38-282.pdf

26. Barba ÁN, Gómez RA. Percepciones acerca de la masculinidad en un grupo de hombres y mujeres del Área Metropolitana de Bucaramanga, Santander, Colombia. Reflexión Política. 2016;18(36):212-23

27. Prado VM, Ramos BE. Narrativas sobre masculinidad, cuerpo y violencia en un grupo de líderes sociales colombianos. Opción. 2020;36(92):939-67.

28. Monárrez LM. Hombre es hombre: diversos ejercicios de masculinidad en un colectivo de jóvenes artistas urbanos en Ciudad Juárez. Transregiones. 2019;1(1):103-23.

29. Arias JJ, Navarro KA, Ortega LD. Agentes educadores y estereotipos sobre masculinidad: reflexiones para la formación de identidades masculinas alternativas. Revista Perspectivas. 2019;4(1):1422. DoI: https://doi.org/10.22463/25909215.1751

30. Ibáñez BG, Vizcarra MT, Ugalde AI. Los recreos, laboratorios para la construcción social de la masculinidad hegemónica. Teoría de la Educación. 2017;29(2):185-209. DoI: http://dx.doi. org/10.14201/teoredu292185209

31. Fonseca-Vindas C. Jóvenes padres costarricenses: cambios y continuidades de la masculinidad tradicional. Revista Espiga. 2019;18(37):14-39. Dor: https://doi.org/10.22458/re.v18i37.2426

32. Bacete R. Las ventajas de la paternidad positiva como un valor emergente, factor de transformación social y de cambio en el paradigma de la masculinidad hegemónica. Hachetetepé. 2017;(15):51-63. DoI: https://doi.org/10.25267/Hachetetepe.2017. v2.i15.6

33. Robaldo M. Aportes en torno a la paternidad y el cuerpo reproductivo masculino a partir de los hallazgos de tres estudios sobre varones y cuidados en Chile y México. Revista Punto Género. 2016;(6):61-72. DoI: https://doi.org/10.5354/07190417.2016.42916

34. Rubio LT. Un análisis sobre nuevas paternidades [Tesis de sociología. Requisito parcial para optar por el título de socióloga]. [Bogotá, Colombia]: Pontificia Universidad Javeriana; 2018.

35. Estrada Montoya JH. Las violencias de género como problema de salud pública: una lectura en clave Bioética. Rev. Colomb. Bioet. 2015;6(1):37-61. https://doi.org/10.18270/rcb.v6i1.1266

36. Gallego-Montes G. Estudios de familia en clave de masculinidades. Estado de la discusión en Colombia. Rev. Latinoam. Estud. Fam. 2018 [internet];10(2):30-50. [citado 2021 mar 22]. Disponible en: https://revistasojs.ucaldas.edu.co/index.php/revlatinofamilia/article/view/3008

37. Berger PL, Luckmann T. La construcción social de la realidad. Buenos Aires: Amorrortu; 2001.
38. Blumer H. El interaccionismo simbólico: perspectiva y método. Hora Nova S. A.; 1982.

39. Cuesta-Benjumea $\mathrm{C}$ de la. La teoría fundamentada como herramienta de análisis. Cultura de los Cuidados. 2006;10(20):136-40. DOI: https://doi.org/10.14198/cuid.2006.20.19

40. Contreras Cuentas MM, Páramo Morales D, Rojano Alvarado YN. La teoría fundamentada como metodología de construcción teórica. Pensamiento y Gestión [internet]. 2020 [citado 2021 mar. 11]; 47(47). Disponible en: https://rcientificas.uninorte.edu.co/index.php/pensamiento/article/view/12342/214421444552

41. Mendoza-Mendoza I, Sanhueza S. Percepciones de equidad de género en las/os futuras/os profesoras/es. Ex æquo. 2018(37):12942. DoI: https://doi.org/10.22355/exaequo.2018.37.09

42. Winfield Reyes AM, Jiménez Galán YI, Topete Barrera C. Representaciones mentales y sociales en la equidad de género. La Ventana. 2017;5(45):186-210.

43. Posada IC, Mendoza Ríos A, Orozco Giraldo IC, et al. Roles de género y salud en mujeres desplazadas por la violencia en Medellín, 2013-2014. Ciencias de la Salud. 2017;15(2):189-202. Dor: https://doi.org/10.12804/revistas.urosario.edu.co/revsalud/a.5754

44. Guber R. La etnografía: método, campo y reflexividad. Buenos Aires: Norma; 2001.

45. Strauss A, Corbin J. Bases de la investigación cualitativa. Técnicas y procedimientos para desarrollar la teoría fundamentada. Medellín: Editorial Universidad de Antioquia; 2002.

46. Donoso T. Construccionismo social: aplicación del Grupo de Discusión en Praxis de Equipo Reflexivo en la Investigación Científica. Revista de Psicología. 2004; 13(1):9-20.

47. González M. Aspectos éticos de la investigación cualitativa. Revista Iberoamericana de Educación. 2002;(29):85-103.

48. Pineda CA, Galindo GK, et al. Validación de un inventario de roles de masculinidad hegemónica en varones colombianos. Diversitas. 2019;15(2):257-70. DOI: https://doi.org/10.15332/22563067.5401

49. Álvarez FJ. Masculinidades en entredicho: resistencia y apoyo de varones adultos de Santiago a la equidad de género [internet] [Tesis para optar al título de Magíster en Estudios de Género y Cultura]. [Santiago de Chile]: Universidad de Chile; 2006 [citado 2018 nov. 10]. Disponible en: http://repositorio.uchile.cl/bitstream/handle/2250/113541/cs39-alvarezf265.pdf? sequence $=1 \&$ isAllowed $=y$

50. Beiras A. A negociação de sentidos sobre masculinidades e paternidades em contextos populares de Florianópolis [internet] [Dissertação (mestrado)]. [Florianópolis, Brasil]: Universidade Federal de Santa Catarina, Centro de Filosofia e Ciências Humanas. Programa de Pós-Graduação em Psicologia; 2007 [citado 2018 nov. 10]. Disponible en: https://repositorio.ufsc.br/bitstream/handle/123456789/90787/242933.pdf? sequence=1\&isAllowed=y

51. Peña JC, Boll V, Arias L. Efecto de la edad en las percepciones de masculinidad en hombres de la región de la Araucanía, Chile. Research on Ageing and Social Policy. 2018;6(2):172-97. DoI:

52. Trujillo Cristoffanini M. Misoginia y violencia hacia las mujeres: dimensiones simbólicas del género y del patriarcado. Atenea (Concepción). 2019;(519):49-64. DOI: http://dx.doi.org/10.4067/ S0718-04622019000100049

53. Carrasco C. La sostenibilidad de la vida humana: ¿Un asunto de mujeres? Mientras Tanto. 2001;(82):43-70.

54. Urra MC. Vivencia subjetiva de la paternidad en hombres de diferentes contextos familiares. Revista Venezolana de Estudios de la Mujer. 2014;19(43):39-85.

55. Mazo AF. Prácticas y significados sobre masculinidad en hombres del municipio de Alejandría-Antioquia [Trabajo de grado para optar al título de trabajador social]. [Medellín, Colombia]: Uni- 
versidad de Antioquia. Facultad de Ciencias Sociales y Humanas. Departamento de Trabajo Social; 2016.

56. Pérez LM. Un panorama en torno a las masculinidades en Medellin 1980-2007 [Trabajo de grado para optar al título de antropóloga]. [Medellín, Colombia]: Universidad de Antioquia. Facultad de Ciencias Sociales y Humanas. Departamento de Antropología; 2008.

57. Velázquez MA. La paternidad en el proyecto de vida de algunos varones de la Ciudad de México. Mneme - Revista Virtual de Humanidades. 2004;5(11):1-14.

58. Burin M, Meler I. Género: una herramienta teórica para el estudio de la subjetividd masculina. En: Varones, género y subjetividad masculina. Buenos Aires: Paidós; 2000.
59. Ospina A. Nuevas masculinidades y cambio familiar: repensando el género, los hombres y el cuidado infantil. Rev. Latinoam. Estud. Fam. [internet] 2020 [citado 2021 mar 20]; 12(1):165-85. Disponible en: http://revlatinofamilia.ucaldas.edu.co/downloads/ Rlef12(1)_10.pdf

60. Salas JM. Hombres que rompen mandatos: la prevención de la violencia. San José, Costa Rica: Lara Segura \& Asociados [internet]; 2005. [citado 2018 nov 12]. Disponible en: https://www. corteidh.or.cr/tablas/r26834.pdf 